\title{
Diseño y desarrollo de simulador de un sistema de almacenamiento de energía mediante sensores piezoeléctricos
}

\section{Design and development of a simulator for an energy storage system using piezoelectric sensors}

\author{
Angélica Calderón ${ }^{l}$, Samantha Ortega ${ }^{l}$, Iveth Moreno ${ }^{1 *}$ \\ ${ }^{1}$ Universidad Tecnológica de Panamá, Centro Regional de Chiriquí
}

\begin{abstract}
Resumen En este trabajo, se diseñó y desarrolló un simulador que permite realizar estudios mucho más profundos en los generadores eléctricos por vibraciones piezoeléctricas; para ello se partió del comportamiento mecánico de un cristal piezoeléctrico sencillo. Se modeló matemáticamente su funcionamiento, obteniendo un circuito eléctrico equivalente al comportamiento mecánico de estos cristales, posteriormente haciendo uso de NI Multisim 14.0, se simuló un sistema cosechador de energía básico. Para obtener datos certeros y observar la eficiencia de la simulación es necesario hacer uso de un cosechador de energía por medio de vibraciones de forma física, por lo que se construyó un dispositivo de almacenamiento de energía mediante piezoeléctricos desarrollado en investigaciones anteriores, a fin de garantizar que sea un buen instrumento de medición para la captura de las variables necesarias. Finalmente se determinó la ecuación que relaciona la fuerza aplicada a los cristales, con la tensión a la salida del cosechador, para así poder aplicar estos datos a las simulaciones desarrolladas, y calcular el porcentaje de error que posee el simulador.
\end{abstract}

Palabras clave Circuito equivalente, cosechador de energía, Multisim, piezoeléctrico, simulador.

\begin{abstract}
In this work, a simulator was designed and developed that allows much more in-depth studies to be carried out in electric generators by piezoelectric vibrations, for this we started from the mechanical behavior of a simple piezoelectric crystal, and its operation was mathematically modeled, obtaining an electrical circuit equivalent to that of mechanical behavior of these crystals, later using Multisim, a basic energy harvesting system was simulated. To obtain accurate data and observe the efficiency of the simulation, it is necessary to make use of an energy harvester by means of vibrations in a physical way, so an energy storage device using piezoelectrics developed in previous research was built, in order to guarantee that is a good measuring instrument for capturing the necessary variables, finally the equation that relates the force applied to the crystals with the tension at the output of the harvester was determined, in order to be able to apply these data to the developed simulations, and calculate the percentage of error that the simulator has.
\end{abstract}

Keywords Equivalent circuit, energy harvester, Multisim, piezoelectric, simulator.

*Corresponding author: iveth.moreno@utp.ac.pa

\section{Introducción}

Dentro del campo de estudio de la generación de energía de forma limpia y sustentable, tenemos a nuestro alcance una gran cantidad de software, simulaciones y aplicaciones que nos permiten analizar la eficiencia de los dispositivos de cosechamiento de energía, tales como paneles solares, turbinas en centrales geotérmicas, entre otros.

Sin embargo, cuando hablamos de generación eléctrica por vibración, en la literatura obtenida durante la investigación, no se encontraron registros de algún software o simulación que permita, de forma eficiente, buscar métodos de mejora de eficiencia de los dispositivos de captación de energía por vibraciones, Sin embargo, existen simulaciones y modelados matemáticos de materiales piezoeléctricos [1], que dan lugar al estudio de simulaciones de generadores de energía mediante vibraciones piezoeléctricas [2].

En este trabajo se busca diseñar y desarrollar un simulador de sistema de almacenamiento de energía por vibración mediante sensores piezoeléctricos obtenidos de buzers [3], utilizando un modelo base de generador eléctrico mediante vibración [1], para el estudio del comportamiento de este sistema de almacenamiento. El trabajo se divide en cuatro etapas principales; en la etapa inicial se estudia el comportamiento mecánico del sensor piezoeléctrico, de forma individual, así como su comportamiento eléctrico ante fuerzas de tensión y compresión. Esta etapa es meramente teórica, de desarrollo matemático y analogías mecánicas y eléctricas a fin de encontrar circuitos equivalentes eléctricamente al comportamiento mecánico del sensor. 
La segunda etapa del proyecto consiste en la utilización del software MULTISIM, para el desarrollo del sistema de almacenamiento de energía mediante sensores piezoeléctricos, con los circuitos equivalentes y parámetros necesarios, determinados en la etapa anterior.

En la tercera etapa se construye un modelo básico de generador eléctrico ya estudiado en investigaciones anteriores, y se realizan pruebas de fuerza y voltaje, con distintos pesos, y a distintas frecuencias, esto con el fin de encontrar una ecuación que relacione la fuerza de compresión proporcionada a los piezoeléctricos, con la tensión a la salida del generador, para poder variar los parámetros de la simulación.

Finalmente, en la última etapa del proyecto, se aplican todos los datos teóricos y matemáticos desarrollados en las etapas anteriores, a las simulaciones del sistema de almacenamiento, y se calcula el porcentaje de error que este posee, con respecto al sistema de almacenamiento de energía mediante sensores piezoeléctricos, en forma física.

\section{Materiales y métodos}

Esta sección fue dividida en tres etapas: La primera etapa consta de tres partes esenciales y consiste en el Análisis del comportamiento físico del sensor piezoeléctrico, tal como se muestra en la figura 1.

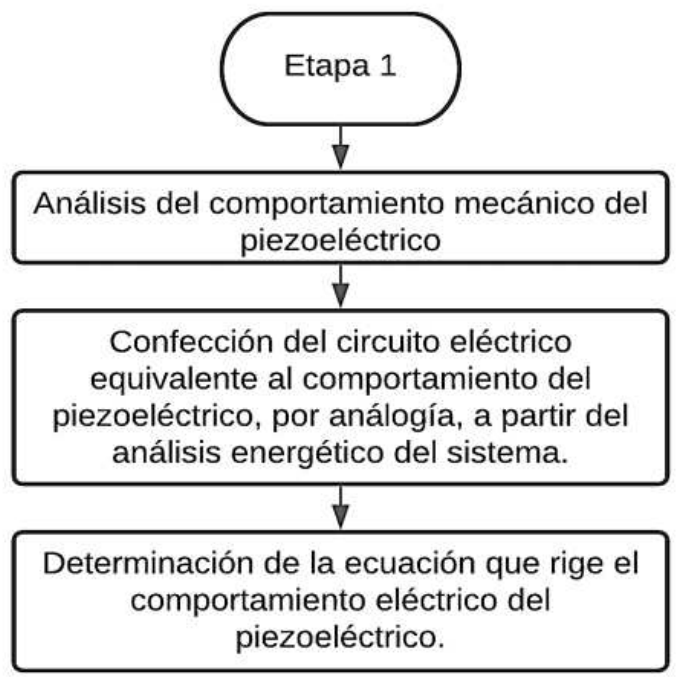

Figura 1. Flujograma de actividades a realizarse en la etapa 1.

La segunda etapa, consiste en el diseño y desarrollo de un simulador, las actividades a realizarse en esta etapa se encuentran descritas en el flujograma de la figura 2.

La tercera y última etapa, tal como se muestra en la figura 3 , consiste en la construcción de un sistema de almacenamiento de energía mediante sensores piezoeléctricos, cuyo diseño y construcción ya ha sido puesta a prueba en investigaciones realizadas por la compañía "Instructables" [1].

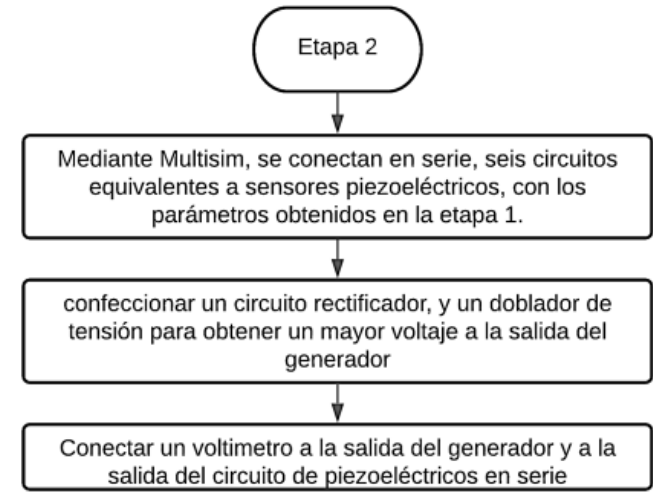

Figura 2. Flujograma de actividades a realizarse en la etapa 2.

Este manual de construcción se encuentra disponible de forma libre. Este generador corresponde a la herramienta de adquisición de datos, ya que, con él, se realizaron pruebas de distintos pesos sobre el sistema, detalladas en la sección 2.3, con el fin de encontrar una ecuación que relacione el voltaje obtenido por el generador en función de la fuerza de compresión aplicada al sistema. Posteriormente, se aplican estos datos y ecuaciones al simulador a fin de comprobar el porcentaje de error de la simulación.

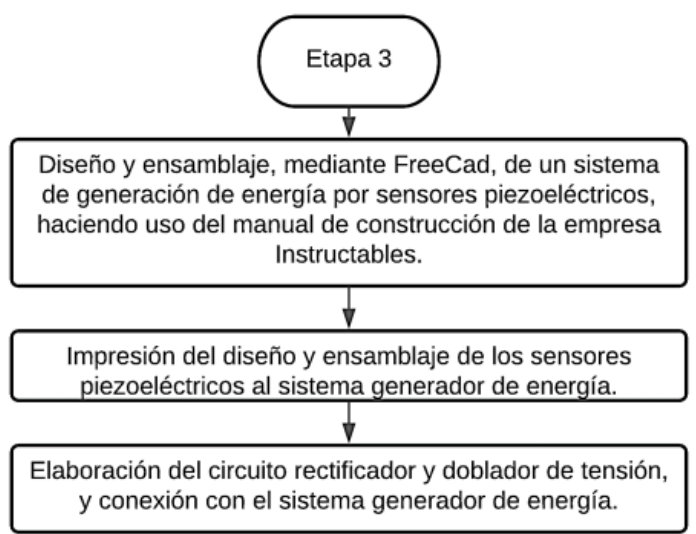

Figura 3. Flujograma de actividades a realizarse en la etapa 3.

\subsection{Análisis del comportamiento mecánico de un sensor piezoeléctrico}

El fenómeno de piezoelectricidad sucede en cristales específicos que, adquieren una polarización eléctrica en su masa al someterse a tensiones mecánicas, provocando una diferencia de potencial y cargas eléctricas en su superficie [2]; las características físicas de los materiales piezoeléctricos se deben, principalmente, a su configuración de atómica.

En el caso de los cristales piezoeléctricos de cuarzo, los átomos de oxígeno y silicio que lo forman se encuentran configurados en un patrón repetitivo; los átomos de silicio poseen cargas positivas y los de oxígeno cargas negativas [3]. 
Comúnmente, al no existir compresión o tensión mecánica, las cargas se dispersan uniformemente a través del cristal, esto sucede cuando se aplica un esfuerzo sobre el material piezoeléctrico, provocando que el átomo se estire o se exprima. $\mathrm{Su}$ ordenamiento cambia ligeramente, este reordenamiento ocasiona una polarización, es decir, las cargas negativas se acumulan en una sección específica del material y las cargas positivas en la sección opuesta. El material puede estar en efecto inverso, lo que implicaría que, en presencia de un campo eléctrico, puede sufrir una deformación en el material; esto implica que la deformación del material también es función del campo eléctrico [1].

El material piezoeléctrico tiene un comportamiento mecánico debido a una fuerza de entrada, que puede ser descrito como un sistema masa-resorte-amortiguador, es decir un sistema de segundo orden, cuyo diagrama se puede apreciar en la figura 4, en la que $m$ es la masa intrínseca en el material piezoeléctrico, $b$ es la constante de viscosidad o el tensor piezoeléctrico y $k$ es la constante de elasticidad, o el módulo de Young [1]. Las señales de entrada y salida del modelo $u(t)$ y $y(t)$ respectivamente, son las que se estudian en la sección 2.3 de este documento.

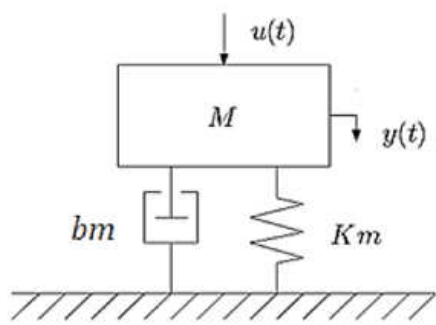

Figura 4. Esquema de modelo mecánico de un transductor piezoeléctrico [4].

$$
\begin{aligned}
& m \cdot \ddot{y}+d \cdot \dot{y}+k y=u(t) \\
& m \cdot \frac{d x^{2}}{d t^{2}}+d \cdot \frac{d x}{d t}+k y=F(t)
\end{aligned}
$$

Por lo tanto, la ecuación que rige el comportamiento mecánico de un material piezoeléctrico es la ecuación (1), y modificando la ecuación para acomodarla a los términos específicos del piezoeléctrico en el dominio del tiempo, se obtiene la ecuación (2), una vez definida esta ecuación que rige el sistema, se puede realizar el análisis energético que nos permitirá realizar la analogía y encontrar un circuito eléctrico equivalente:

- La función de entrada de la fuerza representa energía en forma de trabajo mecánico, que es análogo a una entrada de energía como una fuente de tensión o corriente.

- La fuerza provocada por el resorte mediante energía potencial elástica es análogo a la energía potencial en forma de campo eléctrico, producida por un condensador.
- La masa se encarga de generar energía cinética de movimiento, su análogo eléctrico es un inductor, que genera energía cinética en forma de campo magnético.

- El amortiguador se encargará de reducir las oscilaciones del sistema, su análogo eléctrico es la resistencia, que cumplirá la función de disipar la energía eléctrica en forma de calor.

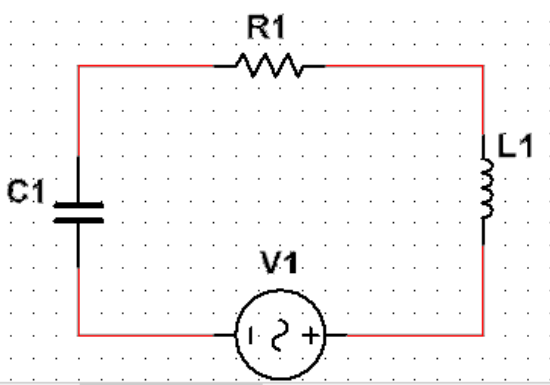

Figura 5. Circuito eléctrico equivalente al comportamiento de un material piezoeléctrico.

$$
L \cdot \frac{d^{2} i}{d t^{2}}+R \cdot \frac{d i}{d t}+\frac{1}{C} i=\frac{V_{0}(t)}{d t}
$$

En la figura 5, podemos visualizar el circuito eléctrico equivalente, que nos permitirá simular el comportamiento mecánico del piezoeléctrico.

La ecuación (3), es la ecuación que rige el comportamiento del circuito. Como podemos ver, la ecuación del circuito eléctrico es equivalente a la ecuación del modelo mecánico, sin embargo, es necesario que en conjunto con el circuito mostrado en la figura 5, se agregue una capacitancia, que se denominará como C2. Esta, es conocida como "capacitancia estática" o "Shunt" que es la suma de varias capacitancias parásitas debidas al propio encapsulado del cristal [5]. Por lo que tomando esto en consideración, y tratando de realizar la simulación lo más apegada a la realidad posible, se modificó el circuito inicial a como se encuentra en la figura 6.

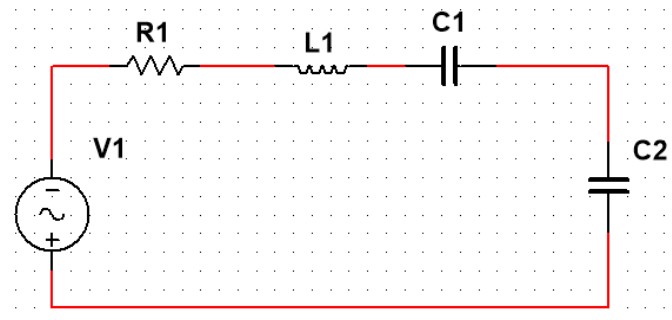

Figura 6. Circuito equivalente de material piezoeléctrico tomando en consideración las capacitancias parásitas.

Para determinar los componentes L1, C1, R1, V1 y C2 del circuito, es necesario tener el DataSheet del material piezoeléctrico a utilizar, en este caso se usará el HC-49/U [6]. Los valores finales extraídos del Datasheet 17 son: $\mathrm{F}=16 \mathrm{MHz}$, $\mathrm{C} 1=30 \mathrm{fF}, \mathrm{R} 1=25 \Omega$ y $\mathrm{C} 2=4.5 \mathrm{pF}$ 
El valor de la inductancia no viene dado por las especificaciones del fabricante, por lo que se puede calcular, sabiendo que es un circuito resonante en serie:

Para que ocurra la resonancia, la reactancia inductiva, debe tener el mismo valor de la reactancia capacitiva, $\mathrm{Xl}=\mathrm{Xc}$ y por lo tanto, al reemplazar los valores de frecuencia de resonancia y capacitancia en la ecuación (4), se obtiene un valor de $\mathrm{L}=3.298 \mathrm{H}$

$$
L=\frac{1}{(2 \pi f)^{2} C}
$$

\subsection{Diseño y desarrollo del simulador en Multisim}

Una vez determinados los valores para el circuito equivalente a un sensor piezoeléctrico, se procede a realizar una conexión de seis elementos piezoeléctricos en serie, en Multisim, con los parámetros obtenidos en el punto 2.1, tal como se aprecia en la figura 7 :

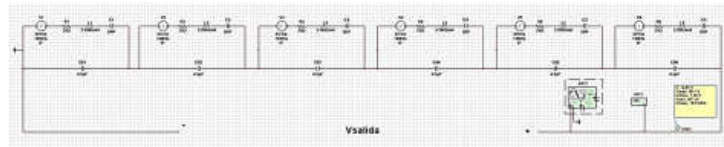

Figura 7. Circuito equivalente de piezoeléctricos en serie en Multisim.

Para poder almacenar la energía proveniente de los materiales piezoeléctricos, es necesario confeccionar un circuito rectificador, adicional a ello se decidió realizar un doblador de tensión para obtener un mayor voltaje a la salida del generador, este circuito, conectado a la salida del circuito de piezoeléctricos en serie, se muestra completo en la figura 8 .

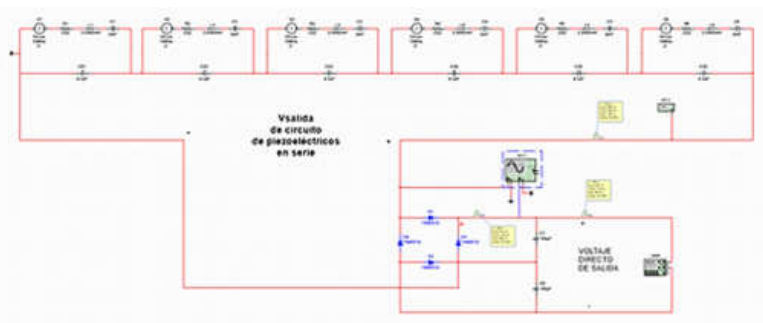

Figura 8. Circuito generador de energía mediante sensores piezoeléctricos.

\subsection{Construcción del circuito físico para realizar las pruebas en el simulador}

Esta sección corresponde al diseño y ensamblaje de un sistema de generación de energía por sensores piezoeléctricos, que ha sido estudiado y puesto aprueba por la empresa "Instructables", el generador base, utilizado en el instructivo que fue realizado por ellos, es mucho más grande y sofisticado [4], por lo que en este proyecto se modificó el sistema para trabajarlo con únicamente seis sensores piezoeléctricos, utilizando el mismo principio de captación de energía.

Se diseñó el sistema de captación de energía por sensores piezoeléctricos, en el software FreeCad, y posteriormente se realizó la impresión del sistema, tal como se muestra en la figura 9, a este sistema, en la parte superior, se le adicionará distintas masas en la parte superior para realizar las pruebas.

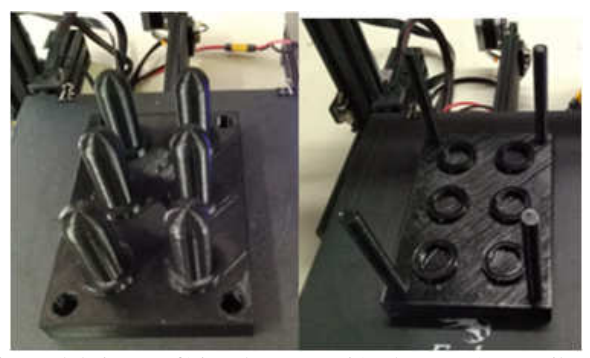

Figura 9. Piezas del sistema físico de generación de energía por vibración.

Posteriormente, como se puede observar en la figura 10, se adhirieron los sensores piezoeléctricos a la base del sistema y se soldaron en serie, posteriormente estos se conectaron al circuito rectificador y doblador de tensión que se puede observar en la figura 11.

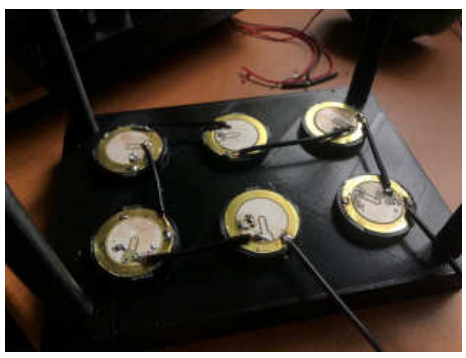

Figura 10. Sistema Físico de generación de energía por vibración con circuito de piezoeléctricos en serie adheridos.

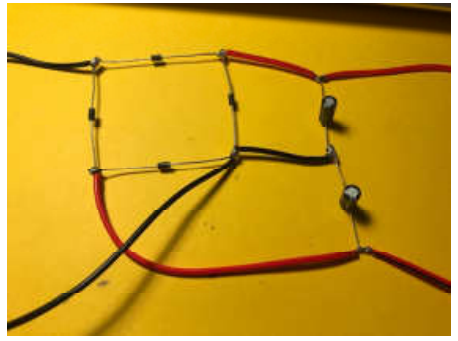

Figura 11. Circuito rectificador y doblador de tensión en físico.

Finalmente se conectó la salida del doblador de tensión, a un multímetro digital, este se encargará de proporcionar el valor final del voltaje a la salida del sistema generador de energía por sensores piezoeléctricos, adicional a ello se utilizó un cronometro para contabilizar el tiempo de funcionamiento para cada prueba, en la figura 12, se puede apreciar el sistema completo, listo para la adquisición de datos, con la masa en la superficie del sistema, esta masa se varió 6 veces, ya que al aumentar a una masa superior a $650 \mathrm{~g}$, los piezoeléctricos corren el riesgo de romperse debido al peso.

El tiempo de recolección de datos para cada prueba es de $120 \mathrm{~s}$, a una frecuencia de $3 \mathrm{~Hz}$, se realizaron 8 pruebas para 
cada masa, con el fin de obtener un valor promedio de voltaje a la salida, que fuera más certero.

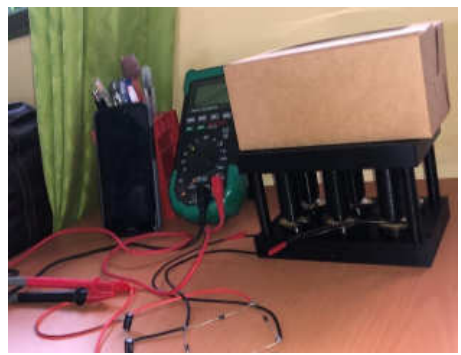

Figura 12. Sistema de generación de energía mediante sensores piezoeléctricos de forma física.

\section{Resultados y discusión}

En esta sección, se divide la captación de resultados y discusión en tres etapas, La primera exclusiva para el análisis de los datos obtenidos en la sección 2.3 del artículo, con el fin de aplicarlos al simulador. En la etapa dos, se realizan la misma cantidad de pruebas del simulador en Multisim, que se hicieron con los datos obtenidos de forma física en la sección 2.3, recogiendo los voltajes de entrada en cada piezoeléctrico y los voltajes de salida del generador. Estos datos son igualmente analizados, para finalmente en la etapa tres, calcular el porcentaje de error del simulador, en relación con el sistema físico.

3.1 Resultados obtenidos con las pruebas mecánicas del generador de energía mediante sensores piezoeléctricos físico

En la tabla 1 se encuentra la tabla de los resultados, es importante destacar que el voltaje que se obtuvo a la salida del generador es un voltaje promedio realizado para ocho pruebas mecánicas para cada prueba, se realizaron un total de 40 pruebas en esta sección, para obtener valores más claros, cada una de estas pruebas fue realizada a una frecuencia de $3 \mathrm{~Hz}$ (oscilaciones del sistema físico), durante dos minutos. La tabla 2, muestra el número de prueba, la masa y el error estándar en las pruebas realizadas.

Tabla 1. Mediciones obtenidas a partir del Sistema generador de energía por piezoeléctricos, construido físicamente

\begin{tabular}{|c|c|c|}
\hline Masa [kg] & Fuerza [N] & Voltaje promedio [mV] \\
\hline 0.205 & 2.01105 & 351.4125 \\
\hline 0.305 & 2.99205 & 456.175 \\
\hline 0.405 & 3.97305 & 623.625 \\
\hline 0.505 & 4.95405 & 1039.25 \\
\hline 0.605 & 5.93505 & 1595.125 \\
\hline
\end{tabular}

Tabla 2. Error Estándar en las mediciones de Tensión obtenidas por el

\begin{tabular}{|r|c|c|}
\hline$\#$ & Masa (kg) & $\begin{array}{c}\text { Error Estándar de la Tensión } \\
\text { Obtenida }\end{array}$ \\
\hline 1 & 0.205 & 23.48473288 \\
\hline 2 & 0.305 & 28.49189108 \\
\hline 3 & 0.405 & 24.28366588 \\
\hline 4 & 0.505 & 25.83325653 \\
\hline 5 & 0.605 & 30.32885236 \\
\hline
\end{tabular}

Se realizaron dos pruebas más, de ocho repeticiones para los primeros dos valores de fuerza, a frecuencias más bajas, realizando 16 pruebas para $1 \mathrm{~Hz}$ y 16 pruebas para $2 \mathrm{~Hz}$ en los valores de fuerza de $2.01105 \mathrm{~N}$ y $2.99205 \mathrm{~N}$, para ambos.

Esto se realizó, para determinar cuál de estos producía el mayor voltaje a la salida, de estas pruebas los resultados fueron valores de tensión mucho más bajos para frecuencias de $1 \mathrm{~Hz}$ y 2Hz. Debido a esto, se decidió realizar las pruebas para los valores de $3 \mathrm{~Hz}$, únicamente; ya que estos eran los óptimos en cuanto a voltaje de salida.

El problema principal con el sistema físico es que es muy difícil hacer que llegue a oscilaciones a más de $3 \mathrm{~Hz}$, por lo que no se realizaron pruebas para valores más altos de frecuencia. En la figura 13 se puede observar los valores de voltaje a la salida del generador, en función de la Fuerza, debido a la naturaleza del gráfico se aplicó una línea de tendencia exponencial, la cual nos dio una regresión de 0.9982 , un valor bastante aceptable.

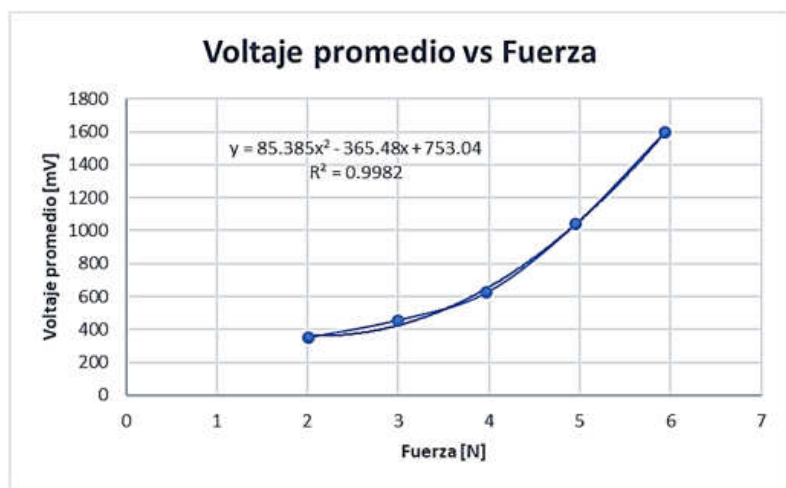

Figura 13. Voltaje promedio obtenido frente a la fuerza aplicada al prototipo.

En la literatura consultada, se encontró estudios de fenómenos de amplitud de voltaje pico-pico versus Fuerza, aplicada en un sensor piezoeléctrico, y este describía una relación lineal; sin embargo, como los datos de voltaje promedio obtenidos en esta investigación, corresponden a el voltaje a la salida del doblador de tensión, tiene más congruencia que el fenómeno sea descrito de forma 
exponencial, tal como lo muestran los resultados en la figura 13.

$$
V_{\text {salida }}=85.385 F^{2}-365.48 F+753.04[\mathrm{mV}]
$$

Al realizar la regresión exponencial en el gráfico 1, se obtuvo la ecuación (5), que corresponde a función del Voltaje de salida en relación con la Fuerza aplicada, para poder introducir los valores de Voltaje en cada piezoeléctrico en relación con la fuerza y ver que tan cercana nos da la simulación a los valores de voltaje de salida del generador obtenidos con el sistema físico es necesario realizar unos cuantos cálculos.

Como los valores que se deben introducir en la simulación deben ser en $\mathrm{V}_{\mathrm{RMS}}$, partimos de la ecuación (6), la cual nos brinda el valor del voltaje RMS del circuito de piezoeléctricos en serie. Debido a que el voltaje a la salida del circuito de piezoeléctricos en serie, es decir, el voltaje ac del circuito, es igual a la suma de los voltajes de cada uno de los sensores piezoeléctricos, se asume que, en las pruebas físicas, todos los piezoeléctricos recibieron uniformemente la misma cantidad de fuerza de compresión sobre sus membranas.

$$
V_{r m s}=\frac{V_{a c}}{\sqrt{2}}=\frac{6 V_{\text {piezo }}}{\sqrt{2}}
$$

La ecuación (6) utiliza el voltaje RMS, proveniente de una fuente sinusoidal pura. Para esta investigación, suponemos que el sistema está siendo excitado por una oscilación armónica [7], debido a que los sistemas normales de generación de energía por vibraciones piezoeléctricas son sistemas con vigas empotradas, o bien, un sistema como el diseñado en la sección 2.2.

Debido a la suposición de una fuente sinusoidal pura, el modelo pierde cierto grado de generalidad, sin embargo, ya que los prototipos de sistemas de generación de energía por medio de vibraciones piezoeléctricas, son pensados para casos específicos en donde su fuente de excitación principal es una fuente controlada de fluido, como en el caso de los recolectores en modelos de boyas [7], o se encuentran montados sobre maquinarias, la suposición de una oscilación armónica, se vuelve altamente representativa

Una vez rectificada esta tensión, por el puente rectificador, tenemos un doblador de tensión, el cual dobla la tensión a la salida del rectificador, por lo que tomando esto en cuenta, se obtiene la ecuación 7, que representa la tensión a la salida del sistema.

$$
V_{\text {salida }}=2 V_{r m s}=\frac{12 V_{\text {piezo }}}{\sqrt{2}}=6 \sqrt{2} V_{\text {piezo }}
$$

Si reemplazamos el valor de la ecuación (7) en (5), obtenemos finalmente la ecuación (8), la cual nos permitirá introducir valores de voltaje a los piezoeléctricos en la simulación en función de la fuerza aplicada al sistema físico.

$$
6 \sqrt{2} V_{\text {piezo }}=85.385 F^{2}-365.48 F+753.04[\mathrm{mV}]
$$

3.2 Resultados obtenidos con las pruebas de voltaje en la simulación del generador de energía mediante sensores piezoeléctricos

Para la obtención de los resultados de la tabla 3 se realizaron 8 mediciones por cada valor de voltaje de entrada de cada piezoeléctrico; esto con la finalidad de contrastar los valores obtenidos mediante la simulación, con los valores obtenidos de forma experimental, con el sistema de forma física. En total se hicieron 40 pruebas, cada una durante un tiempo de $120 \mathrm{~s}$ y una frecuencia de $3.3 \mathrm{~Hz}$, la relación existente entre el voltaje obtenido a la salida del generador, con relación a los voltajes de entrada de los piezoeléctricos, se puede observar en el figura 14, donde se puede ver, que a diferencia del figura 13, su línea de tendencia es un poco más lineal, esto resulta contradictorio a lo esperado según el figura 13 y la sección 3.1, sin embargo esto puede ser debido al aumento del error en la simulación, a medida que aumentan los valores de fuerza; esta situación se puede mejorar, tomando en cuenta la fuente oscilatoria de excitación del sistema, como una función que se adapte mejor a la oscilación excitatriz de este, durante las pruebas físicas.

Tabla 3. Voltaje obtenido vs voltaje de entrada por medio de la simulación del circuito equivalente

\begin{tabular}{|c|c|c|c|}
\hline $\begin{array}{c}\text { Tiempo } \\
{[\mathbf{s}]}\end{array}$ & $\begin{array}{c}\text { Frecuencia } \\
{[\mathbf{H z}]}\end{array}$ & $\begin{array}{c}\text { Voltaje de } \\
\text { entrada }[\mathbf{m V}]\end{array}$ & $\begin{array}{c}\text { Voltaje } \\
\text { obtenido } \\
{[\mathbf{m V}]}\end{array}$ \\
\hline 120 & 3.3 & 64 & 349.748 \\
\hline 120 & 3.3 & 75 & 438.098 \\
\hline 120 & 3.3 & 114 & 433.869 \\
\hline 120 & 3.3 & 180 & 702.256 \\
\hline 120 & 3.3 & 274 & 1042 \\
\hline
\end{tabular}

\section{Voltaje obtenido vs Voltaje de entrada}

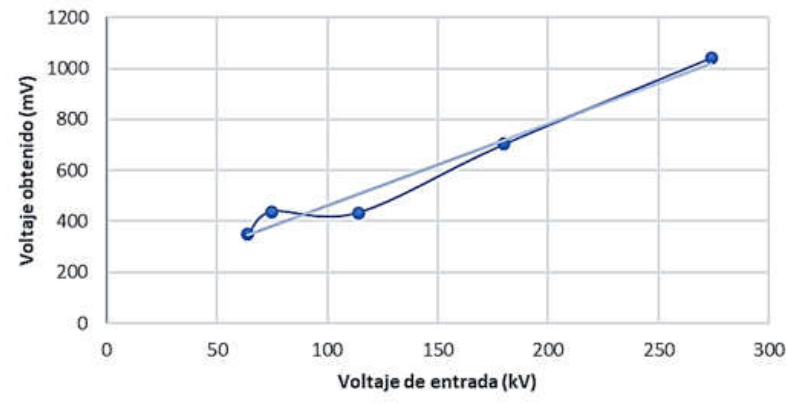

Figura 14. Voltaje promedio obtenido frente al voltaje de entrada de cada piezoeléctrico por medio de la simulación. 
3.3 Comparación de resultados obtenidos en pruebas físicas y de simulación del generador de energía mediante sensores piezoeléctricos

Luego de haber recopilado los valores del voltaje obtenido para ambas metodologías se procedió a calcular el porcentaje de error entre ambos resultados obtenidos para cada caso mediante la ecuación (9), donde tomamos como valor experimental el voltaje obtenido por el sistema físico y como valor aproximado el voltaje obtenido mediante el simulador.

$$
\% \text { Error }=\frac{\mid \text { Valor Aproximado-Valor Experimental } \mid}{\text { Valor Exacto }} \times 100 \%
$$

Tabla 4. Porcentaje de error obtenido luego de utilizar ambas metodologías

\begin{tabular}{|c|c|c|}
\hline $\begin{array}{c}\text { Voltaje obtenido - } \\
\text { Sistema Físico } \\
{[\mathbf{m V}]}\end{array}$ & $\begin{array}{c}\text { Voltaje obtenido - } \\
\text { Simulador [mV] }\end{array}$ & $\begin{array}{c}\text { Porcentaje } \\
\text { de error } \mathbf{( \% )}\end{array}$ \\
\hline 351.4125 & 349.748 & 0.47 \\
\hline 456.175 & 438.098 & 3.96 \\
\hline 623.625 & 433.869 & 30.43 \\
\hline 1039.25 & 702.256 & 32.43 \\
\hline 1595.125 & 1042 & 34.68 \\
\hline
\end{tabular}

Como se puede observar en la figura 15 , al realizar la comparación del voltaje obtenido con ambas metodologías ambas curvas tienen la misma forma, sin embargo, a pesar de que inician con valores cercanos se obtuvieron valores que divergen al aumentar la fuerza de compresión en los piezoeléctricos, esto implica que el simulador funciona de forma eficiente para valores pequeños de fuerza, sin embargo, diverge para valores muy grandes; esto puede deberse a la poca precisión y falta de generalidad en la ecuación (6), ya que se utilizó la suposición de que el sistema es excitado por una oscilación armónica.

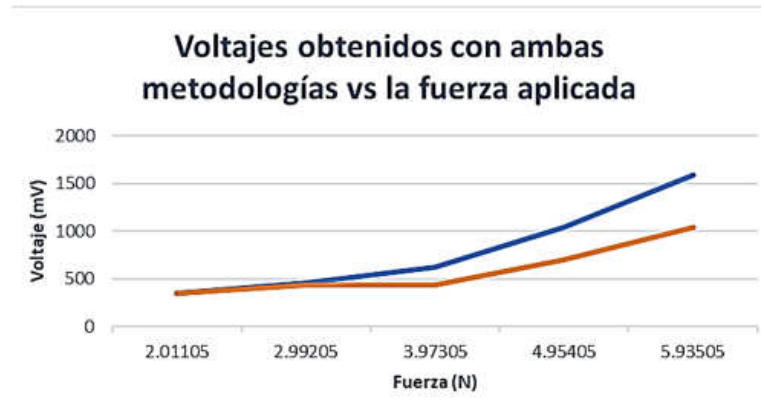

Voltaje obtenido - Físico [mV] $\longrightarrow$ Voltaje obtenido - Simulador [mV]

Figura 15. Comparación de voltaje obtenido a la salida del generador de forma física, con el voltaje obtenido a la salida del generador en la simulación, para los mismos valores de fuerza.

\subsubsection{Discusión}

Este trabajo abre las puertas a la investigación e innovación. Dado que, al contar con una herramienta de análisis de datos para generadores eléctricos por vibración se puede lograr la creación de nuevos prototipos para la cosecha de energía y la implementación de estos. Esto garantiza que el sistema será más sencillo y fácil de construir, sin tantos gastos de inversión en el proyecto, en cuanto a materiales y pruebas.

Además, la utilización del software Multisim, implica que la simulación sea fácil de imitar, modificar y mejorar, lo que presenta una gran ventaja ante la elaboración de un simulador sin un software base, este programa nos arroja datos bastante cercanos a los deseados.

Dentro de las principales limitaciones de este proyecto se encuentra la limitación al momento de comparar la eficiencia del simulador haciendo uso de cristales piezoeléctricos provenientes de buzzer, esto limita a los usuarios de la simulación a tener que utilizar ese tipo de cristales para la elaboración de su sistema de generación eléctrica por vibración, o limita el tipo de proyecto a los cuales está dirigido, ya que estos datos de porcentajes de error solo aplican para cristales piezoeléctricos estandarizados, de $5 \mathrm{~V}$, para buzzer.

Otra limitante importante, fue la asunción de la función excitatriz del sistema, como una oscilación Armónica. Esto provoca perdida de generalidad en la simulación, al igual que pérdida de precisión en la ecuación utilizada para encontrar los valores de voltaje que se introducen a Multisim, un importante trabajo a futuro consiste en generar una función excitatriz del sistema que cumpla matemáticamente con el comportamiento oscilatorio provocado en las pruebas físicas.

\section{Conclusiones}

Luego de la culminación de cada una de las etapas del proyecto logramos concluir lo siguiente:

- La simulación del sistema de recolección y almacenamiento de energía por medio de sensores piezoeléctricos supone una gran ventaja y un avance en la generación de energía asequible, ya que por medio de esta se pueden analizar y probar distintos métodos que mejoren la eficiencia del sistema, sin necesidad de realizar un gasto en cuanto a materiales y pruebas.

- La eficiencia del simulador disminuye a medida que aumentan los valores de fuerza de compresión en los sensores piezoeléctricos, esto puede deberse a la suposición de la función excitatriz del sistema, ya que, al tomarla como una función armónica, el sistema pierde generalidad y la ecuación tiende a ser imprecisa en cuanto a la descripción matemática del fenómeno que provoca la excitación del sistema.

- Un posible trabajo a futuro es el cálculo de los porcentajes de error del simulador con respecto a las pruebas físicas, en distintos tipos de materiales piezoeléctricos, como membranas piezoeléctricas, sensores piezofilm u otros tipos de cristales con las mismas propiedades de piezoelectricidad, o con 
distintos sistemas, cuya excitación sea una oscilación no armónica.

\section{AGRADECIMIENTOS}

Este trabajo de investigación fue realizado gracias a la colaboración del profesor José Serracín en el proceso de diseño e impresión del sistema generador de energía por sensores piezoeléctricos, e igualmente extendemos un agradecimiento al profesor Omar Aizpurúa, por aportar los conocimientos de analogía eléctrica por análisis energético, que ayudaron en gran medida al diseño de la simulación.

\section{REFERENCIAS}

[1] O. Borda, L. Mendoza. "Modelo matemático de un generador piezoeléctrico", Trabajo fin de máster, Facultad de Ingeniería Electrónica, Universidad Santo Tomás, Bogotá D.C., 2019.

[2] A. Gomez-Casseres, "Visualización de magnitudes piezoeléctricas usando COMSOL Multiphysics», en I Congreso Internacional de Visualización de Datos, Bogotá, 2014, pp. 2-8.

[3] C. A. Chacon, J. D. Cortés, D. A. Giral, R. R. Romero. "Piezoelectricidad en un BUZZER", Tecnura, vol. 16, pp.112120, octubre 2012.

[4] Instructables Circuits. Piezoelectric energy harvester, 2013. [En línea]. Disponible:
https://www.instructables.com/Piezoelectric-Energy-Harvester/ [Accedido: 17-Julio-2020].

[5] A. I. Diaz, "Materiales Piezoeléctricos: Calculo y simulación del circuito equivalente de un Cristal de Cuarzo", presentado en la Universidad Tecnológica Nacional, Bahía Blanca, Argentina, 2015.

[6] Alldatasheet. HC49U Datasheet (PDF) - Fox Electronics. 2003 [En línea]. Disponible: https://pdf1.alldatasheet.com/datasheetpdf/view/239759/FOX/HC49U.html [Accedido: 20-Julio2020]

[7] C. F. Quiróz. "Comparación y prueba de modelos para generación de electricidad por vigas piezoeléctricas enfocado a pequeños dispositivos electrónicos", Trabajo fin de grado, Departamento de Ingeniería Mecánica, Universidad de Chile, Santiago de Chile, 2015.

[8] A. M. Malmcrona. "Aplicaciones del efecto piezoeléctrico para la generación de energía", Trabajo fin de grado, Universidad Carlos III de Madrid, Leganés, 2018.

[9] D. Alonso, M. Vázquez, F.J. Jiménez, J. de Frutos. "Optimización de piezoeléctricos comerciales para su uso en sistemas de Energy Hasvesting", Elsevier España, vol.54 pp.247-253, Nov. https://doi.org/10.1016/j.bsecv.2015.09.001

[10] M. Vázquez, F.J. Jiménez, J. de Frutos, "Modelización de materiales piezoeléctricos como generadores de energía", Bol. Soc. Esp. Ceram. Vidr. Vol. 51, pp.25-36, 2012. 